\title{
Men who have sex with men (MSM) living with HIV in India: Challenges and opportunities for community support
}

\section{ABSTRACT}

This paper focuses on one component of a larger study exploring the perceptions of people living with HIV in India. The paper reports on the findings of a small investigation centring on the experiences of a selected population of men who have sex with men (MSM) in India who are living with HIV. Two focus groups were facilitated with 22 MSM in Andhra Pradesh, India, to explore a range of challenges for men living with HIV within the Indian context. Qualitative data were analysed through descriptive qualitative content analysis. Findings emphasised the impacts of experiences of discrimination and marginalisation as well as the significance of the role of community support as a critical factor in enhancing the lives of HIV affected MSM within India.

Keywords: Men who have sex with men (MSM), HIVIAIDs, challenges, community perceptions, community support, stigma, India.

\section{Introduction}

It is widely documented that men who have sex with men (MSM) are stigmatized worldwide, with homosexual sex deemed a punishable offence in 82 countries globally (Baral, Trapence, Motimedi, Umar, lipinge, Dausab F, et al. 2009; Beyrer C, Baral SD, van Griensven F, Goodreau SM, Chariyalertsak S, Wirtz AL, et al. 2012; UNAIDS 2013). One of the most significant current challenges in the prevention of HIV is addressing the HIV epidemic amongst MSM in resource-limited settings (De Cock, Jaffe and Curran 2012). Within the Indian context, it is estimated that there are 2.1 million people living with HIV (PLHIV) (National AIDS Control Organization (NACO 2016). Although the reduction in the prevalence of HIV over the past decade in India has been significant, achieving an estimated peak of $0.38 \%$ between $2001-2003$ continuing at the level of $0.34 \%$ and $0.28 \%$ in 2012 , reducing to $0.26 \%$ in 2015 (NACO 2016), this reduction primarily represents the decline in heterosexual populations, with HIV prevalence among MSM remaining largely static (NACO 2016).

Given the stigma associated with the practices of MSM, as well as laws banning anal intercourse and entrenched cultural norms that limit marriage options for men to women, the prevalence rates of HIV among MSM have been difficult to document (Solomon, Mehta, Srikrishnan et al. 2015; Gupta, Mehta, Godbole, et al. 2006; Herget 2006; Asthana and Oostvogels 2001). In a study by Solomon et al. (2015) examining HIV amongst MSM in India across twelve cities for example, results highlighted significant diversity in prevalence and incidence rates. The findings also suggest burgeoning epidemics in locations where HIV has previously been less prevalent (Solomon, Mehta, Srikrishnan et al. 2015).

This paper reports on the findings of a study focusing on the lived experiences of MSM living with HIV in two locations in India. The study explored the perceptions of a group of participants recruited for the 
investigation to examine the challenges associated with living as MSM as well as managing the impacts of HIV. The paper gives emphasis to experiences of marginalisation across the cohort interviewed for the study. Exploration of the role of community support is also examined as a means of assisting MSM in living with HIV. Recommendations for future research and practice to promote awareness of the needs of HIV-affected MSM, including investigation of cogent community based intervention frameworks will be explored in order to guide policy and practice within the Indian context that addresses issues associated with this marginalised population.

\section{MSM and HIV: The Indian Context}

The occurrence of HIV epidemics in MSM internationally is steadily increasing (Beyrer, Baral, Griensven, Goodreau, Chariyalertsak, Wirtz and Brookmeyer 2012; Thomas, Mimiaga, Kumar, Swaminathan, Safren and Mayer 2011). However, there are significant gaps in knowledge about HIV for MSM globally, due to the stigma associated with this population (Solomon, Mehta, Srikrishnan et al. 2015; Beyrer, Wirtz, Walker, Johns, Sifakis, and Baral 2011; Gupta, Mehta, Godbole, et al. 2006; Khan 2004). The reported estimate of HIV prevalence for MSM in India is 4.3 per cent (NACO 2016).

Same sex behaviour has been illegal in India since 1860, with Section 377 of the Indian Penal Code rendering sex with persons of the same gender punishable by law (Government of India Ministry of Law and Justice 2017). As a result, MSM experience a range of complex challenges including criminalisation, discrimination and marginalisation (Beattie, Bhattacharjee, Suresh, Isac, Ramesh and Moses 2012). Further, such challenges have adverse affects on the effectiveness of HIV prevention strategies across the country (Thomas, Mimiaga, Mayer, Closson, Johnson, Menon, Mani, Vijaylakshmi, Dilip, Betancourt \& Safren, 2012; Thomas, Mimiaga, Kumar, Swaminathan, Safren and Mayer 2011; Mawar, Sahay, Pandit and Mahajan 2005).

There are various sub-groups of MSM in India including Kothis (who display feminine behaviours/characteristics and are usually receptive partners in anal intercourse), Panthi or Danga (who exude masculine characteristics and are insertive participants in anal sex), Double Deckers (men who have sex with men and women) and Alis or Hijras (transgendered people) (Kumta, Lurie, Weitzen, Jerajani, Gogate, Row-kavi, Anand, Makadon and Mayer 2010; Thomas, Mimiaga, Mayer, Johnson, Menon, Chandrasekaran, Murugesan, Swaminathan and Safren 2009).

A range of studies has been facilitated in India and other contexts to examine the utility of community based interventions focusing on HIVIAIDs for MSM populations. However, many of these interventions give emphasis to primary prevention approaches (Panos Institute 1992; Schoef 1993; Asthana and Oostvogels 1996; Campbell and Mzaidume 2001; Basu, Jana, RotheramBorus, Swendeman, Lee, Newman and Weiss 2004; Chattopadhyay and McKaig 2004; Cornish and Ghosh 2007; Evans and Lambert 2008) rather than focusing on systems of community-based support for PLHIV. Further, existing HIV preventative measures for MSM in India are primarily limited to condom distribution, HIV prevention education, voluntary HIV counselling and testing, 
and treatment of sexually transmitted infections (STIs) (Thomas, Mimiaga, Kumar, Swaminathan, Safren and Mayer 2011).

A recent study by Thomas, Mimiaga, Mayer et al., (2011) examined the perceptions of 55 MSM about HIV preventative interventions currently available in Chennai, India. The findings of the study revealed that the majority of participants were in favour of intervention programs that extended beyond HIV and STI risk reduction, giving focus to the broader psychosocial needs of HIV-affected MSM. A study by Verma, Shekhar, Khobragade et al. (2010) examined the efficacy of a HIV prevention program to support female sex workers and MSM in four Indian states. The results indicated that facilitation of the program resulted in efficacious outreach activities at a district level (Verma, Shekhar, Khobragade et al. 2010). In another study by Sivaram, Zelaya, Srikrishnan, Latkin, Go, Solomon and Celentano (2009) key findings centred on the significance of membership within community groups and supportive networks in reducing fear of transmission, shame and judgement associated with living with HIV. Such membership ultimately increased the social capital of beneficiaries (Sivaram, Zelaya, Srikrishnan, et al. 2009).

In a study by Larmar (2016) the perceptions of 11 community workers in the implementation of an intervention providing psychosocial support for PLHIV (including MSM) in Northern India were investigated. The study highlighted the positive impacts of a community support model for PLHIV where better psychosocial adjustment for individuals living with HIV involved increasing community acceptance and integration and management of the illness. Kabore et al. (2010) examined the utility of an intervention combining community-based support and medical assistance for PLHIV in sub-Saharan Africa. The outcomes of the study highlighted the merits of community-based support in improving health outcomes for PLHIV. Further, a study by Jana, Basu, Rotheram-Borus and Newman (2004) investigated the benefits of a community-based HIV prevention and treatment program facilitated in Calcutta, India. The findings of the study demonstrated the program's application to settings beyond the community under examination.

While a small sample of studies has been identified exploring community-based approaches to supporting MSM in India, Further research needs to be undertaken to understand the lived experiences of MSM including the role of community-based support to assist MSM in managing the challenges of living with HIV in India. The present study was facilitated to extend the limited knowledge about MSM and HIV in India as well as to establish the effectiveness of community-based support in assisting individuals to manage the challenges of living with HIV.

\section{Method}

The study was conducted over a three-week field trip to Andhra Pradesh, India utilising a qualitative methodology. The method for the investigation was informed by social constructionism as the philosophical framework undergirding the study. Burr (2003) recognises the unique application of social constructionism to social research emphasising that "...there can be no final description of the world, and reality may be inaccessible or inseparable from our discourse about it; all knowledge is provisional and contestable, and accounts are local and historically/culturally 
specific" (p.156). Further, social constructionism gives focus to the social dimensions associated with the psychology of individuals and communities (Gergen, 2009), rendering it a suitable philosophical framework to guide the chosen methodology. A qualitative approach was deemed to be a suitable method of enquiry given that the phenomenon of interest focused on the experiences of MSM in managing the daily challenges associated with living with HIV, experiences that are shaped and understood through social interaction. It was critical that the research methods employed for the study elicited results that gave voice to the unique and often under-represented voices of MSM living with HIV in India.

Three primary research questions served to guide the investigation. These included:

1) What do MSM with HIV understand about living with HIV, including management of their illness?;

2) What are some of the dominant community perceptions associated with MSM and HIVIAIDs?; and

3) What forms of support are available to MSM living with HIV and what is their perceived effectiveness?

A facilitated focus group discussion approach was chosen to seek answers to the three research questions. This method of enquiry allows for a more collaborative, nuanced approach between the researcher and participant whereby individuals recruited for a study can provide rich information associated with their experience, in contrast to quantitative studies, which use structured questionnaires that are limited to the exploration of a series of variables.

Two focus groups were facilitated in two geographical locations that were comparative in predominant demographic characteristics within communities in Andhra Pradesh. Individuals were recruited for the study through word of mouth from outreach workers employed within two local nongovernment organisations (NGOs). The individuals recruited for the study were drawn from semi-rural locations, with all participants living in lower socio-demographic contexts. Consideration of contextual factors such as location and demographic profile of participants was critical to the study to ensure that the specific population under investigation was clearly represented. The first focus group consisted of $12 \operatorname{MSM}(n=12)$ and the second group, $10 \mathrm{MSM}(n=10)$, both living in rural areas of Andhra Pradesh, India. The mean age of the full sample group was $(n=35)$.

Prior to the commencement of the focus groups, all potential participants were provided with an overview of the research including: the themes to be investigated; method of data collection (focus group discussion); explanation and assurance that all responses would be kept strictly confidential and no identifying information would be recorded during the data collection process to ensure the anonymity of all participants; that participation in the research was voluntary and that any participant could withdraw from the research at any time without penalty; and, that the results of the research were intended to benefit the MSM HIVIAIDS affected communities through contribution to the field of HIVIAIDS/Social Development research focusing on factors that support communities living with HIVIAIDs in India. Full ethical approval for the study was obtained from the researcher's university. 
In preparation for the focus groups, the researcher developed a series of in-depth questions in consultation with a local coordinator of an NGO working with HIVIAIDs-affected men. The development of these questions in collaboration with a local specialist working within the communities under investigation was one method that ensured the reliability and trustworthiness of the data being collected. Throughout the process of formulating questions the researcher and local consultant considered the major themes of significance for the study to ensure the focus of the investigation was maintained. Themes included: participant awareness of HIVIAIDs including management of the illness; community perceptions of HIVIAIDS including issues of marginalisation and stigma; and attitudes and key considerations associated with MSM with HIVIAIDs more generally.

Prior to the facilitation of each focus group, the researcher, with the assistance of the NGO coordinator, received signed consent from all participants. The focus groups commenced with an introduction that included orientation to the focus group methodology employed for the study as well as explanation of the conditions associated with each individual's voluntary participation, including that all participants had the right to not respond to any of the focus group questions and to withdraw from the group discussion at any time.

Each focus group was facilitated over a two-hour period. The utilisation of the questions was instrumental in engaging participants in a semistructured discussion to explore some of the key dimensions associated with living with HIVIAIDs highlighted above.

\section{Results}

\section{Research Question One}

For the first research question: What do MSM with HIV understand about living with HIV, including management of their illness?, a summary of focus groups responses are outlined below:

Knowledge about HIV before receiving treatment. Prior to treatment, the majority of participants had heard about HIV; that it was a disease, but no one knew anything about it other than that it was a serious illness. Four participants had no knowledge about HIV prior to diagnosis. One participant had substantial knowledge about HIV given that his parents were both living with HIV. However, he knew little about treatment. Some participants acknowledged that they held misconceptions about HIV and its spread. For example, one participant believed that you could not make any physical contact with someone with HIV due to the risk of contamination. Another stated he had understood that stepping onto urine that was HIV contaminated would lead to contracting the disease. Others were aware of the social stigma associated with the condition. One participant mentioned that you could become infected by the cough of a HIV carrier. Another participant had experienced seeing a HIV positive individual being removed from their village and was concerned that the same thing would happen to him.

Access to Treatment. The majority of participants indicated that they were introduced to a range of HIV treatment options through either: a) a local NGO that supports PLHIV; b) an NGO supporting MSM; or c) government hospital services. A number of participants indicated that they first learned about HIV 
treatment through outreach workers/social workers located in local NGO services. Others communicated that government hospital support workers were instrumental in assisting them to access services. Integrated Counselling Testing Centres (ICTC) were also identified as instrumental in offering services such as free post-test counselling. Three participants reported that they were able to access health related literature that discussed HIV treatment.

Current Knowledge about HIV following Initial Treatment. In terms of knowledge about HIV following initial contact with medical personnel, the majority of participants indicated that their knowledge about HIV had increased. Participants all became aware of the conditions associated with the transmission of HIV, personal challenges associated with living with HIV in India, current treatment approaches such as ART, implications for failing to adhere to treatment protocols, understanding the significance of CD4 counts and safe sex practices. Participants also indicated that following initial treatment they developed awareness about the role of nutrition in managing their condition, as well as avoiding other infections including STIs. A resounding theme that emerged through this line of enquiry was that despite the HIV diagnosis, individuals could lead normal lives through ongoing health care.

Approaches to manage living with HIV. A range of responses about managing life with HIV emerged through the focus group discussion that bare similarities to those responses derived from the previous question. For example, participants mentioned the important role of good nutrition and personal hygiene in managing HIV. Psychosocial support was also highlighted as a significant aspect of managing the challenges of living with HIV. Group meetings with other PLHIV were identified as particularly useful. Such interaction provided a source of hope and a sense of belonging. Ongoing support from medical professionals was also raised as part of the discussion about HIV management, including adhering to ART treatment as well as other lifestyle choices such as regular exercise, yoga and mindfulness activities.

\section{Research Question Two}

For the second research question: What are some of the dominant community perceptions associated with MSM and HIVIAIDs?, the following findings were deemed significant:

Family/community knowledge about the individual's HIV status. Approximately $50 \%$ of participants' families were aware of each respective individual's diagnosis, while the remaining $50 \%$ of families had not been informed. For those individuals whose families had been informed, they reported that levels of familial support increased as their families developed greater knowledge about HIV. It was interesting to note that all participants had chosen to keep their diagnosis hidden from the broader community due to underlying fear of discrimination.

Factors influencing non-disclosure of HIV status. There was a range of reasons associated with choosing not to disclose information about an individual's HIV status. The overarching concern stemmed from anxieties associated with the stigma attached to PLHIV. One participant mentioned concern that his children would be ashamed of him and his wife may abandon him. Another individual expressed concern that his wife may be moved to suicide. One of the participants indicated that he'd had sexual contact with 
some of his neighbours and lived in fear that, if they were to find out about his diagnosis, they would become angry and attempt to harm him. The majority articulated an overarching concern about family and community members stigmatising them, which may lead to them experiencing social rejection that would ultimately culminate in them losing the love, respect and acceptance of people who were important to them.

Experiences of stigmatisation. Part of the focus group discussion emphasised individual's experiences of stigmatisation. It was interesting to note that a number of participants revealed that they had experienced discrimination from some members of the gay community because of their HIV status. One individual relayed a story about being beaten by a number of straight men when he was waiting at a bus stand. The beating was a result of the men learning of his HIV status and their concern that he would spread the disease throughout the community. Another participant told a story about how one man was murdered as a result of people within the community discovering that he had HIV. Another individual described how his wife became enraged by the knowledge that he had contracted HIV and that she "felt cheated by him". She moved away from him and took the children with her. She has, since the initial separation, denied him access to his children.

Attitudes towards people who are gay and HIV positive. A focus on community attitudes towards gay people and PLHIV was considered. The following responses were highlighted during the discussion. A number of participants' family members experienced shame knowing that they had a relative who identified as either gay or HIV positive. The majority acknowledged the common perception that gay people are effeminate. Others indicated that heterosexual people make fun of them and reject them based on their sexual orientation. One participant had experienced blackmail from people. It was communicated that some individuals within the community perceive that gay people are neither male nor female. Others articulated that people act aggressively or distance themselves from PLHIV. For example, participants agreed that auto-drivers would tell people to stay away from them. Others indicated that government hospitals would not perform surgical procedures once they find out about an individual's HIV status. The groups agreed that there is more discrimination because many gay men are feminine in character and it is assumed that they have contracted HIV through homosexual activity. Another participant described the experience of one young individual who was HIV positive and who ended up experiencing serious mental health issues, which led to him living on the street because of his fear of people knowing about his status. He was described as having gone mad.

Responses to HIV status from the LGBTIQ community. Many participants experienced solidarity within the LGBTIQ community in relation to their sexual orientation and HIV status. Mention was given to the role of NGOs in helping individuals to improve both their physical and psychological health. Many participants shared the experience of bringing their wives for counselling that culminated in bringing about positive change. However, as noted in an earlier section, some instances of discrimination about a person's HIV condition occurred within the LGBTIQ community. One participant articulated that sometimes when he was walking with his boyfriend, members of the LGBTIQ community would mock him and say he was a "plus" meaning that he was HIV 
positive. Another participant relayed the story of a young boy who would visit one of the NGO's supporting gay men who are HIV positive. He was deaf and dumb and uneducated. He knew very little about the disease. A TV channel invited him for an interview and assured him that that they would not broadcast his identity. They ended up disclosing his identity, resulting in him losing his job and moving to an isolated village to find work because his reputation was ruined. All participants agreed that greater acceptance occurred once individuals got to know PLHIV personally.

Fears about living with HIV. Many of the focus group participants experienced a range of fears associated with living with HIV. A primary fear shared by all participants was the experience of rejection from family and community. Others expressed concerns about their chances of survival given some of the limitations associated with the work they could do to support their families due to issues associated with their illness. Many individuals feared for the future of their children given the concern that they may die as a result of their HIV status. One individual expressed concern about infecting his wife, a second expressed concern about his wife attempting to commit suicide, while a third shared his fears about dying. Finally, one individual indicated that he had no fears about the condition of HIV itself; his primary fear was the experience of discrimination as a result of the disease.

\section{Research Question Three}

For the final research question that guided the investigation: What forms of support are available to MSM living with HIV and what is their perceived effectiveness?, the following responses were considered significant.

Government services to support PLHIV. A range of government services was identified by the participants that served to support PLHIV. One individual commenced this part of the discussion by emphasising that there is not much government help for PLHIV. However, there appears to be more help for heterosexuals. Other members of the group confirmed this. He added that once medical personnel became aware that he was MSM they discriminated against him. Participants indicated that pension schemes were offered for some people who are HIV positive once they had completed a year of ART medication. Others were eligible for ration cards for rice and other food items. The group highlighted that ART medication was freely available from government hospitals. A number of participants mentioned that free testing for HIV and other STIs were also available, as well as free pre- and post- test counselling. The group also mentioned that free condoms were available through government agencies. It was interesting to note that no mention was given to the provision of government services that would offer psychosocial support for individuals living with HIV.

Medication to manage living with HIV. As indicated earlier, the provision of ART medication at a government level was instrumental in providing support to manage individuals' illness. Of note, not all participants were taking ART medication because their CD4 counts were above 350 . However, five participants confirmed taking ART medication. All participants expressed a strong level of awareness about ART medication and understood that government services provided access to medication free of charge. Further, all participants were aware of the significance of adhering to the strict 
protocols associated with ART treatment to arrest the ongoing development of HIV and to promote improved health.

NGO support to assist PLHIV. Within both focus groups, overwhelming support was given to the role of NGOs in providing psychosocial support for PLHIV. As reflected in documented responses in earlier sections, the following information affirmed the support provided by NGO services for PLHIV. The majority of participants spoke about the role of local NGOs in orientating them to HIV treatment and overall health management to personally address issues associated with the condition. Integral to NGO support services was the function of outreach workers in assisting PLHIV within local community settings. Such workers were instrumental in introducing individuals to the NGO service and in providing an initial orientation to managing HIV. It was perceived that a major function of the NGOs was to provide support for PLHIV to improve their physical health and assist in their psychosocial adjustment to living with HIV. As mentioned in an earlier section, counselling services for PLHIV and their extended family members was a useful approach in supporting families and instilling hope. Another significant area of support facilitated by local NGOs involved the organisation of peer support and group-based meetings to assist individuals in managing the challenges of living with HIV. This approach contributed to individual's experiencing a sense of belonging and hopefulness.

Medical support, including management of ART as well as psychoeducational opportunities focusing on health and lifestyle were also notable contributions that NGOs were making in supporting individuals in their adjustment to living with HIV.

\section{Discussion}

The results of the study emphasise the prevalence of discrimination for MSM living with HIV in India. While it is important to recognise that much is being done within India to reduce marginalisation of PLHIV it is clear that further work is necessary to mitigate the deleterious impacts of discriminatory attitudes and behaviours for PLHIV more generally and for MSM specifically. It was interesting to note that evidence of discrimination also existed within the LGBTIQ community. Such trends suggest that, although the Indian Government have instigated a range of community awareness strategies in an attempt to educate the general population about HIV and AIDs, more work needs to be done to address misconceptions about PLHIV. In this way, experiences of discrimination may be reduced which will ultimately improve the lived experiences of HIV-affected individuals.

A commonly reported affect of discrimination within the participant group was the experience of fear of some societal repercussions for living with HIV, such as rejection from family and community members, unemployment etc. Many participants indicated that they feared for their physical and/or psychological safety because of the daily realities of rejection and marginalisation for MSM with HIV. This culminated in many individuals choosing to avoid disclosing their HIV status within their immediate communities, including close family members.

Despite evidence of discrimination and marginalisation, it was encouraging to see how the role of government services have assisted MSM 
with HIV in the provision of health care management, including the distribution of free ART, medical testing, pension schemes and counselling services. However, for the majority of participants, there was a shared sense that government agencies were not generally supportive of MSM with HIV. Many articulated that they experienced some form of discrimination from government health employees. The results highlight that, while government support is an important factor in educating participants about HIV-related health issues including treatment, management of lifestyle factors such as diet and regular exercise and safe sex practices, further attention is needed to address government agency interactions that may marginalise HIV-affected MSM. Despite some evidence of discriminatory attitudes and behaviours inherent in government-related services, a range of government support services were instrumental in improving the general health and wellbeing of all individuals recruited for the study, emphasising the importance of the provision of easily accessible, non-discriminatory government-based services to improve the quality of life of PLHIV.

Another important finding of the study centred on the role of community support in improving the lives of MSM with HIV. All participants gave voice to the sense of connectedness and belonging that resulted from interaction with various NGOs and community agencies. Evidence of the impacts of a range of supportive interventions to assist HIV-affected MSM highlighted the integral role that NGOs can play in the provision of psychosocial support for PLHIV. For all participants, local NGOs were instrumental in increasing access to services that positively influenced individual health management, including specific HIV medical treatment. Further, the function of NGO outreach personal was integral to the widening of community access to HIV support services and reducing perceived barriers to treatment. Of significant importance was the facilitation of a range of services that centred upon the individual's psychosocial adjustment to living with HIV. Counselling services and the coordination of peer support were highlighted as beneficial to managing the challenges of living with HIV in India.

\section{Recommendations and Limitations}

The reported study has a number of recommendations to guide future practices in the delivery of services for MSM with HIV in India. First, it is clear that both government and NGO services within India must give priority to raising awareness about the issues experienced by MSM and PLHIV more broadly as a means of increasing community support and reducing discrimination for this marginalised population. Second, an increased focus on ongoing government led initiatives, such as health care support for MSM with HIV would assist in improving the lives of this group more generally. Finally, a focus on enhancing services within India that provide community based psychosocial support for MSM and PLHIV would serve to facilitate holistic and timely intervention approaches to improve the lives of individuals managing the challenges of living with HIV in India.

A number of limitations were evident within the study that should be noted. First, the small participant sample and the two locations within which the study was facilitated limit the generalisability of the results for contexts that differ both culturally and demographically from the population under 
investigation. Future research should be directed towards exploring the lived experiences of MSM with HIV using larger sample populations and a greater range of contexts. Second, the participant sample received support from locally based government and NGO services, limiting the perceptions of participants to these services only. Other studies should focus on the examination of alternative services in other geographical locations. Finally, the component of the study focusing on community support only gave attention to specific intervention strategies utilised by the identified services. Other intervention frameworks aiming to support PLHIV and MSM in particular should be considered for future research to determine the most efficacious means of improving the lives of MSM with HIV in India and in other international contexts.

\section{Conclusion}

This paper has reported on the findings of a small qualitative study focusing on the experiences of MSM living with HIV in India. The results of the investigation emphasise the daily challenges for HIV affected MSM, including the stigma and marginalisation directed towards MSM populations within Indian society. The study highlights the range of government and nongovernment services for PLHIV that provide support to promote physical health and psychological wellbeing. A key finding of the investigation emphasised the important role of NGOs in facilitating community-based support to assist individuals in the psychosocial adjustment to living with HIV. Such support included the strengthening of social connection and community acceptance as a means of improving the lives of a population who have traditionally experienced stigmatisation and ostracism from broader Indian society.

\section{Bibliography}

Annual Report 2015-16. New Delhi: Department of AIDS Control; National AIDS Control Organisation; 2010.

Asthana, S., and Oostvogels, R. 1996. "Community Participation in HIV Prevention: Problems and Prospects for Community-Based Strategies Among Female Sex Workers in Madras." Social Science and Medicine, 43: $133-148$.

Asthana, S., and Oostvogels, R. 2001. "The Social Construction of Male 'Homosexuality' in India: Implications for HIV Transmission and Prevention." Social Science and Medicine, 52:707 - 721.

Baral, S., Trapence, G., Motimedi, F., Umar, E., lipinge, S., Dausab, F., and Beyer. 2009. "HIV Prevalence, Risks for HIV Infection, and Human Rights Among Men who have Sex with Men (MSM) in Malawi, Namibia, and Botswana." PLoS One, 4 (3): 1 - 8. 
Borus, M.J., Swendeman, D., Lee, S-J., Newman, P. and Weiss, R. 2004. "HIV Prevention Among Sex Workers in India." Journal of Acquired Immune Deficiency Syndromes, 36 (3): 845 - 852.

Beattie, T.S.H., Bhattacharjee, P., Suresh, M., Isac, S., Ramesh, B.M., and Moses, S. 2012. "Personal, Interpersonal and Structural Challenges to Accessing HIV Testing, Treatment and Care Services Among Female Sex Workers, Men who have Sex with Men and Transgenders in Karnataka State, South India." Journal of Epidemiol Community Health, 66: $42-48$.

Beyrer, C., Baral, S.D., van Griensven, F., Goodreau, S.M., Chariyalertsak, S., Wirtz, A.L, et al. 2012. "Global Epidemiology of HIV Infection in Men who have Sex with Men.' Lancet, 380: 367 - 377.

Beyrer, C., Wirtz, A.L., Walker, D., Johns, B., Sifakis, F., and Baral, S. 2011. "The Global HIV Epidemics Among Men who have Sex with Men. Washington, D.C: The World Bank.

Burr, V. 2003. Social Constructionism. East Sussex: Routledge.

Campbell, C., and Mzaidume, Z. 2001. Grassroots Participation, Peer Education and HIV Prevention by Sex Workers in South Africa. American Journal of Public Health, 91 (12): 1978 - 1986.

Chattopadhyay, A., and McKaig, R.G. 2004. Social Development of Commercial Sex Workers in India: An Essential Step in HIVIAIDS Prevention. AIDS Patient Care and STDs, 18 (3): 159 - 168.

Cornish, F., and Ghosh, R. 2007. The Necessary Contradictions of 'Community-Led' Health Promotion: A Case Study of HIV Prevention in an Indian Red Light District, Social Science and Medicine, 64: 496 507.

De Cock, K.M., Jaffe, H.W., and Curran, J.W. 2012. The Evolving Epidemiology of HIVIAIDS. AIDS, 26: 1205 - 1213.

Evans, C., and Lambert, H. (2008). Implementing Community Interventions for HIV Prevention: Insights from Project Ethnography, Social Science and Medicine, 66: 467 - 478.

Gergen, K. (2011). The self as social construction. Journal of Psychological Studies, 56(1), 108-116.

Gupta, A., Mehta, S, Godbole, S.V., Sahay, S., Walshe, L., Reynolds, S.J., Ghate, M., Gangakhedkar, R.R., Divekar, A.D., Risbud, A.R., Mehendale, S.M., and Bolinger, R.C. 2006. Same-Sex Behavior and High Rates of HIV Among Men Attending Sexually Transmitted Infection Clinics in Pune, India (1993-2002), Journal of Acquired Immune Deficiency Syndrome, 43, (4): 483 - 490.

Government of India Ministry of Law and Justice. Act No.45 of 1860, Indian Penal Code, Chapter XVI, Section 377 (Last modified April 27). ncw.nic.in/acts/THEINDIANPENALCODE1860.pdf

Herget, G. 2006. India: UNAIDS Claims Law Criminalizing Homosexuality Hinders HIV Prevention. HIV AIDS Policy Law Review, 11: 35 - 36.

Jana, S., Basu, I., Rotheram-Borus, M.J., and Newman, P.A. 2004. The Sonangachi Project: A Sustainable Community Intervention Program. AIDS Education and Prevention, 16, (5): 401 - 414.

Kabore, I., Bloem, J., Etheredge, G., Obiero, W., Wanless, S., Doykos, P., Ntsekhe, P., Mtshali, N., Afrikaner, E., Sayed, R., Bostwelelo, J., Hani, A., Moshabesha, T., Kalaka, A., Mameja, J., Zwane, N., Shongwe, N., 
Mtshali, P., Mohr, B., Smuts, A., and Tiam, A. 2010. The Effect of Community-Based Support Services on Clinical Efficacy and HealthRelated Quality of Life in HIVIAIDS Patients in Resource-Limited Settings in Sub-Saharan Africa. AIDS Patient Care and STDs, 24, (9): $581-594$.

Khan, S. 2004. MSM and HIVIAIDs in India, Naz Foundation International, 1 23.

Larmar, S.A. 2016. Perceptions of Community Workers in the Provision of Psychosocial Support for Individuals Living with HIVIAIDs in India.

European Journal of Business and Social Sciences, 4, (10): 61 - 72.

Mawar, N., Sahay, S., Pandit, A., and Mahajan, U. 2005. The Third Phase of HIVIAIDS Pandemic: Social Consequences of HIVIAIDS Stigma and Discrimination and Future Needs. Indian Journal of Medical Research, 122: $471-484$.

National AIDS Control Organization and Institute of Health and Family Welfare. Annual HIV Sentinel Surveillance Country Report. Last modified June 14.2006 http://www.naco.gov.in/upload/Surveillance/Reports\%20\&\%20Publicati on/HIV\%20Sentinel\%20Surveillance\%202006 India\%20Country\%20R eport.pdf.

National Institute of Medical Statistics and National Aids Control Organisation. Technical Report: India HIV Estimates. Last modified June 14. 2010: http://naco.gov.in/upload/Surveillance/Reports\%20\&\%20Publication/Te chnical\%20Report\%20India\%20HIV\%20Estimates\%202010.pdf.

Panos Institute. 1992. The Hidden Cost of AIDS: The Challenge of HIV to Development. London: Panos Institute.

Schoeof, B.G. 1993. AIDS Action-Research with Women in Kinshasa, Zaire, Social Science and Medicine, 37: 1401 - 1413.

Sivaram, S., Zelaya, C., Srikrishnan, A.K., Latkin, C., Go, V.F., Solomon, S., and Celentano, D. 2009. Associations Between Social Capital and HIV Stigma in Chennai, India: Considerations for Prevention Intervention Design, AIDS Education and Prevention, 21, (3): 233 - 250.

Solomon, S.S., Mehta, S.H., Srikrishnan, A.K. Vasudevan, C.K., Mcfall, A.M. Balakrishnan, P., Anand, S., Nandagopal, P., Ogburn, E., Laeyendecker, O., Lucas, G.M., Solomon, S., and Celentano, D.D. (2015). High HIV Prevalence and Incidence Among Men who have Sex with Men (MSM) across 12 Cities in India, AIDs, 29 (6): 723 - 731.

Thomas, B., Mimiaga, M.J., Kumar, S., Swaminathan, S., Safren, S.A., and Mayer, K.H. 2011. HIV in Indian MSM: Reasons for a Concentrated Epidemic and Strategies for Prevention, Indian Journal of Medical Research, 134: 920 - 929.

Thomas, B., Mimiaga, M.J., Mayer, K.H. Closson, E.F., Johnson, C.V., Menon, S., Mani, J., Vijaylakshmi, R., Dilip, M., Betancourt, T., and Safren, S.A. 2012. Ensuring it Works: A Community-Based Approach to HIV Prevention Intervention for Men who have Sex with Men in Chennai, India, AIDS Education and Prevention, 24 (6): 483 - 499.

United Nations Joint Programme on HIVIAIDS. Global report: UNAIDS report on the Global AIDS Epidemic. Modified at June 7. 2013: Available at: http://www.unaids.org/sites/default/files/en/media/unaids/contentass 
ets/documents/epidemiology/2013/gr2013/UNAIDS Global Report 20 13 en.pdf.

United Nations Joint Programme on HIVI AIDS. Global report: UNAIDS reports on the Global AIDS Epidemic. Modified at June 14. 2010: Available at: http://issuu.com/unaids/docs/unaids globalreport 2010/1?e=0.

Verma, R., Shekhar, A., Khobragade, S., Adhikary, R., George, B., Ramesh, B.M., Ranebennur, V., Mondal, S., Patra, R.K., Srinivasan, S., Vijayaraman, A., Paul, S.R., and Bohida, N. 2010. Scale-up and Coverage of Avahan: a Large-Scale HIV Prevention Programme Among Female Sex Workers and Men who have Sex with Men in Four Indian States, Sexually Transmitted Infections, 86, (1): 76 - 82. 